\title{
Expérience migratoire et exil social dans la migration congolaise : de l'enjeu d'un corpus littéraire en sciences sociales. Biyaoula, Mabanckou et N'Sonde
}

\author{
Migratory Experience and Social Exile in the Congolese Migration: Of Using a \\ Literary Corpus in Social Sciences. Biyaoula, Mabanckou and N'Sonde \\ Experiencia migratoria y exilio social en la migración congoleña: del desafío de \\ un corpus literario en ciencias sociales. Biyaoula, Mabanckou y N'Sonde
}

\section{Mélanie Pénicaud}

\section{OpenEdition Journals}

\section{Édition électronique}

URL : https://journals.openedition.org/remi/8584

DOI : 10.4000/remi.8584

ISSN : $1777-5418$

\section{Éditeur}

Université de Poitiers

\section{Édition imprimée}

Date de publication : 1 mars 2017

Pagination : 65-89

ISBN : 979-10-90426-30-6

ISSN : 0765-0752

\section{Référence électronique}

Mélanie Pénicaud, «Expérience migratoire et exil social dans la migration congolaise : de l'enjeu d'un corpus littéraire en sciences sociales. Biyaoula, Mabanckou et N'Sonde », Revue européenne des migrations internationales [En ligne], vol. $33-n^{\circ} 1$ | 2017, mis en ligne le 01 mars 2019, consulté le 14 avril 2022. URL : http://journals.openedition.org/remi/8584; DOI : https://doi.org/10.4000/remi.8584 


\title{
Expérience migratoire et exil social dans la migration congolaise : de I'enjeu d'un corpus littéraire en sciences sociales. Biyaoula, Mabanckou et N'Sonde
}

\author{
Mélanie Pénicaud ${ }^{1}$
}

\section{Introduction}

À la faveur de la tendance actuelle d'ouverture des sciences sociales à la littérature (avec des approches parfois très différentes, voir par exemple Grignon et Passeron, 1989 ; Brown, 1992 ; Lepenies, 1995 ; Privat, 1994 ; Cnockaert, Privat et Scarpa, 2001 ; Heinich, 2005 ; Barrère et Martuccelli, 2009 ; Lahire, 2011 ; Bensa et Pouillon, 2012), le champ des migrations internationales étend les possibles de ses sources et objets. Cette inclinaison est elle-même inscrite dans une réflexion sur les rapports entre sciences sociales et écriture menée dès l'avènement de ces premières, ainsi que dans une volonté moins ancienne de dynamiser le dialogue entre les disciplines. En ce sens, de récents travaux en sciences sociales portant sur les migrations tendent à intégrer la littérature dans leurs préoccupations (voir notamment De Gourcy, 2013 ; Schor, 2013)². Néanmoins, cette orientation marque surtout de nombreuses approches venues de la critique littéraire qui, sur des questions liées à la migration, intègre depuis plus longtemps encore des éléments et des analyses de nature sociologique ou anthropologique, qu'elle croise désormais parfois avec les vues de chercheurs en sciences sociales (voir par exemple Montandon, 2006 ; Montandon et Pitaud, 2006 ; Gras, 2009 ; Ghidina et Violle, 2014 ; Lalagianni et Moura, 2014 ; Larangé, 2014 ; Misrahi-Barak et Raynaud, 2014 ; Barbour et al., 2015). Dans le contexte des migrations africaines, cette préoccupation est particulièrement présente dans les départements d'études francophones états-uniens (Cazenave, 2003 ; Moudileno, 2001, 2003 et 2006 ; Thomas, 2013) tout en apparaissant dans la production française (Mongo-Mboussa, 2002 ; Albert, 2005). S'inscrivant dans

1 Anthropologue, Docteure de I'Université de Poitiers, MIGRINTER, MSHS, Bâtiment A5, 5 rue Théodore Lefebvre, TSA 21103, 86073 Poitiers cedex 9 ; melaniepenicaud@gmail.com

2 On note également le cycle de rencontres Non-lieux de l'exil entrepris par Alexandra Loumpet-Galitzine et Alexis Nouss qui prolonge les réflexions menées antérieurement au sein du groupe Poexil, ainsi qu'un numéro d'Hommes et Migrations intitulé Écrire la migration (2014). 
cette filiation et s'attachant à une démarche de recherche compréhensive de la migration, cet article interroge le hiatus disciplinaire qui caractérise la dénomination de l'expérience, à savoir que, lorsque le littéraire traitera de préférence de "l'exil ", le chercheur en sciences sociales parlera plutôt de " migration ". Ainsi, à partir d'un corpus composé de différents écrits, nous différencierons et confronterons les notions d'expérience migratoire et d'expérience exilique pour mieux comprendre comment elles s'agencent.

L'analyse ici proposée se trouve au creux de deux enquêtes de terrain, dont l'une a été réalisée auprès de ressortissants iraniens (Pénicaud, 2012 et 2015) et I'autre avec des ressortissants congolais (Pénicaud, 2017). Or, si dans le cas des entretiens compréhensifs menés avec les Iraniens, les termes d'exil et d'exilé(e) (s) apparaissaient très souvent, aucun des quarante Congolais et Congolaises interrogés ne les a spontanément évoqués. Serait-ce à dire que l'exil ne fait pas partie de l'expérience de ces migrants ? S'intéresser à ce qu'exprime sur ce point la littérature semble donc nécessaire : quelle place l'exil, thème littéraire par excellence (Talahite-Moodley, 2007 ; Bonn, 2000), y trouve-t-il donc ? Comment s'exprime-t-il alors ? Ces lignes mettent en avant, pour le contexte congolais, différentes manières d'être et de se sentir exilé, et ce, tant dans la société dite " de départ " que celle " d'accueil ". Néanmoins, dans les limites de cet article, nous ne faisons pas ici dialoguer ces deux types d'expression de et sur la migration (soit orale et écrite) : les lignes qui suivent s'appuient exclusivement sur un corpus d'œuvres littéraires dans lequel sont analysées les convolutions des expressions de l'exil et de la migration.

Choisies principalement pour l'audience qu'elles ont reçue ${ }^{3}$, ces œuvres s'étalent sur une quinzaine d'années: L'Impasse de Biyaoula est parue en 1996, Le Cour des enfants léopards en 2007, suivie du Silence des esprits (2010) de N'Sonde, publiée un peu après Black Bazar (2009) de Mabanckou 4 . Or, leur lecture amène à avancer une définition de l'exil relativement éloignée de celle que l'on retrouve habituellement dans les études sur la migration, soit un exil social, et ce malgré des contextes différents ; car si le personnage principal de L'Impasse incarne un migrant congolais "lambda " sur le territoire français et celui de Black Bazar également, à ceci près qu'il y est aussi écrivain, celui du Silence des esprits est un ancien milicien sans-papiers et celui du Cour des enfants léopards un jeune homme ayant grandi dans un quartier de la banlieue parisienne. Cependant, c'est vers le même paradigme d'exils à la fois multiples, mais surtout de nature sociale, dépassant l'idée de l'appartenance

3 Publié aux éditions Présence africaine, L'Impasse a reçu le Grand Prix littéraire de I'Afrique noire en 1996. Le Cœur des enfants léopards, paru chez Actes Sud, a été récompensé du prix Senghor de la création littéraire et du prix des Cinq Continents de la francophonie en 2007. Si Black Bazar, publié chez Seuil, n'a, de même que le Silence des esprits, reçu aucune distinction littéraire, il a néanmoins bénéficié d'un certain succès au sein de la critique et en librairie et a été traduit en plusieurs langues.

4 La production de la littérature de migration congolaise en France s'étend, à notre connaissance, de 1986 à nos jours. Si, aux côtés d'Henri Lopès ou de Gabriel Mwèné Okoundji, Alain Mabanckou, Wilfried N'Sonde et Daniel Biyaoula figurent parmi les plus célèbres, $d$ 'autres auteurs à la production relativement moins abondante ou moins remarquée sont à relever, parmi lesquels et pour ne citer qu'eux, Itoua Ndinga, Arian Samba, ou Victor Foutou. La production féminine est quant à elle plus discrète, avec toutefois les créations de Jeannette Balou-Tchichelle, ou encore de Liss Kihindou, par exemple. 
(Bianchi, 2005), s'opposant à l'idée doxatique et restrictive d'un exil synonyme d'une " migration forcée ", d'un bannissement ou d'une fuite " ailleurs " avec ses variations nostalgiques autour du pays d'origine, que nous conduisent ces lectures. La possibilité d'exils multiples fait ici référence à celle d'un double exil intérieur - mais non moins social - chez l'homme noir migrant, Africain ou Antillais, dans un contexte postcolonial : à l'exil "chez soi ", hérité de la "situation coloniale " (Balandier, 1951) - doublé pour l'Antillais d'une mémoire traumatique de l'esclavage -, s'ajoute un autre exil que forge l'expérience de la migration dans le pays "d'arrivée ". Il serait en outre possible de lire, à côté de ces exils de nature sociale, un exil compris à partir de la métaphore végétale du déracinement et que forgerait l'expérience migratoire. Toutefois, cette dernière idée d'un migrant déraciné et d'un sentiment d'exil se référant à ce type de métaphore ne retient pas ici notre attention : en effet, comme l'ont déjà montré et souligné Malkki (1995) ou Simon-Lorière à propos des réfugiés - position que nous étendons ici aux migrants en général - la conception réifiante et " récurrente du réfugié déraciné, employant une métaphore botanique, s'appu[ie] sur une conception sédentaire et stato-centrée [voire ethno-centrée], et impliqu[e] l'idée que le déplacement est une anomalie " (Simon-Lorière, $2013: 554)$. L'exil social est donc à comprendre dans le lien qui unit l'individu à la société dans laquelle il vit à l'instant $t$. L'exil social est, dans son rapport avec la société de laquelle il est exclu, un exil au présent.

\section{Assumer la pluridimentionalité du concept d'exil pour affiner la compréhension des expériences migratoires}

Les limites de cet article ne nous permettent pas de développer autant qu'il le faudrait la pluridimentionalité de l'exil. II est néanmoins nécessaire de mentionner les grandes orientations qui participent de la richesse et de la complexité du terme avant de s'attarder sur la dimension sociale de la notion, mise en évidence par notre propos.

L'exil pourrait en effet trouver un espace plus large en sciences sociales, permettant, sinon de bousculer un lexique de la migration aujourd'hui affaibli (Wievorka, 2013 ; Nouss, 2015), du moins de compléter et d'enrichir les analyses $s^{\prime} y$ rapportant. Nous ne pouvons aujourd'hui que constater la crise qui frappe le lexique de la migration (ibid.) résultant, à la fois, de catégories préconstruites, de la politisation et de la médiatisation de ses objets, et par là, de la connotation de nombreux termes s'y rapportant. Pour certains, parler en termes de " migrant " et de " migration " appellerait ainsi plutôt une approche politique, administrative ou démographique, quand utiliser le terme d' " exil " permettrait de mieux prendre en compte la dimension subjective, expérientielle de la migration (Nouss, 2015). Cependant, le concept d'expérience migratoire, déjà existant, n'est-il pas déjà là pour en rendre compte ? Ne doit-on pas alors plutôt penser l'expérience exilique au prisme de l'expérience migratoire ?

Les sciences sociales ne sont malgré tout probablement pas en reste de l'appauvrissement du terme d'" exil " : alors que la notion requiert un regard transdisciplinaire, géographes et sociologues ont " dissous " (Wievorka, 2013) I'exil dans le champ d'étude des migrations internationales. II apparaît en effet, sous 
la plume de nombre de ces chercheurs, réduit à l'expression de la migration forcée. Ce constat oblige qui s'intéresse à l'exil en tant que " condition " (Nouss, $2015)^{5}$ et situation réflexive à procéder avant tout à un effort de définition. Car enfin, le succès et le potentiel littéraire de l'exil qui attestent de l'universalité du thème et de l'expérience sont probablement aussi en grande partie responsables du délaissement du terme. Or, cet universel n'est-il pas lui-même fécond pour la pensée scientifique, en particulier anthropologique?

La "potentialité heuristique unique, et pourtant négligée " ${ }^{6}$ de l'exil, possible recours venant combler les apories des recherches sur les migrations, doit avant tout se saisir dans les principales dimensions qui la composent et en font un objet polysémique : topos littéraire - notamment romantique - très ancien, objet poétique, politique et philosophique, l'exil est à la fois bannissement et départ non librement consenti, mais est aussi travaillé par de nombreux philosophes pour sa portée universelle et positive (Bianchi, 2005), solitaire ou collectif (pensons au galout par exemple), il tend parfois en ce sens vers la notion de diaspora. L'exil comme expérience peut ainsi se penser dans les oppositions qui le fondent : celles de l'actif et du passif, du positif et du négatif, de l'intérieur et de l'extérieur. Ces antagonismes marquent en effet son évolution, tandis que l'étymologie du terme souligne déjà cette bipolarisation : l'exsilium exprime à la fois le lieu d'exil, l'exil et le bannissement, mais également, par le verbe qui s'y rapporte (exsilio), I'action de bondir, de sauter, de sortir hors de, aussi bien que l'action de s'élancer, s'élever. La négativité qui encadre le terme d'exil aujourd'hui dans le langage commun et tel qu'il est également saisi par les sciences sociales sous la forme de migration dite "forcée ", n'est donc qu'une construction historique (Bordes-Benayoun, 2006 : 189) qui néglige les travaux philosophiques et polarise leur attention sur le pays d'immigration, tandis que I'exil est aussi " ce qui nous fait croire à la seconde fois ou à la seconde chance " (Bianchi, 2005 : 8-9), à la résilience, ailleurs. Cette bipolarisation, ce balancement ou cette "discontinuité " (Saïd, $2002: 140$ ) sont constitutifs de l'exil. Pour Nouss (2015), I'exil se définit d'abord comme " condition et conscience ", nommant, inspiré par Derrida, mais au risque d'une réification, " exiliance " ce " noyau existentiel " incarné par l'exil dans la migration. Appuyé sur cette première définition de Nouss, notre propos conçoit l'exil dans une triade condition, conscience et réflexivité, la notion de réflexivité soulignant que l'exil est aussi objet de création, littéraire et artistique.

Enfin, penser et définir l'exil comme condition, conscience et réflexivité, c'est penser une expérience appuyée sur un mouvement de retrait par rapport à soi. L'exil donnant la distance nécessaire pour pouvoir rendre compte de ce qui est vécu, l'expérience de l'exil n'est alors que le récit et le discours mêmes de cette expérience. Car penser l'exil, c'est se penser soi-même, ou du moins tel est le geste de l'exilé. Celui qui fait l'expérience de l'exil s'interroge sur sa place dans le monde : quelle est-elle et quelle est celle de I' " autre " ? La mobilisation des travaux d'Arendt, et principalement l'évocation du concept de " désolation " nécessairement appelé par l'idée d'une " place " en ce monde " reconnue et

5 Chercheurs qui, généralement, ne sont pas directement issus au champ des migrations internationales, mais plutôt de la psychologie ou de la littérature.

6 Citation tirée du site Internet du Collège d'études mondiales : http://www.collegeetudesmondiales.org/fr/content/s\%C3\%A9minaire-lexp\%C3\%A9rience-de-lexil 
garantie par les autres »(Arendt, $2005: 307)$, interroge et précise en ce sens la compréhension des expériences exiliques.

Chez Arendt (2005), la " désolation " dépasse la solitude et l'isolement, se comprenant dans un sentiment de dépossession, à la fois de son identité propre et de toute réalité. Pour Arendt, "La désolation, fond commun de la terreur, essence du régime totalitaire, [...] est étroitement liée au déracinement et à I'inutilité dont ont été frappées les masses modernes depuis le commencement de la révolution industrielle et qui sont devenus critiques avec la montée de l'impérialisme à la fin du siècle dernier, et la débâcle des institutions politiques et des traditions sociales à notre époque. Être déraciné, cela veut dire n'avoir pas de place dans le monde, reconnue et garantie par les autres ; être inutile cela veut dire n'avoir aucune appartenance au monde " (Arendt, 2005 : 306-307). Aussi peut-on, dans le contexte migratoire, considérer que la désolation puisse suivre comme précéder le départ.

Sur ce point, les travaux de Weil (1949) et son propre développement du concept de " déracinement " sont ainsi à rapprocher de la position d'Arendt. Dans L'enracinement, Weil substitue la notion d'obligation à celle de droit en dressant le portrait des nécessaires obligations que toute communauté se doit de fournir à tout homme. Si l'enracinement est de ces nécessités, il n'est pas pour autant à lire à l'aune d'une métaphore végétale, car les racines de cette notion weilienne ne sont pas celles du sol, mais celles des sociétés dans lesquelles les hommes vivent : l'enracinement, chez Weil, procède du social. Lorsque ces racines sont rompues, lorsque la famille ou l'état s'effondrent ou éclatent, lorsque la transmission ne se fait plus, lorsque que travail devient aliénant, le déracinement est là, affectant l'" âme humaine " et le privant de son nécessaire enracinement. Si c'est à l'aune de ces deux notions que nous lisons principalement la dimension sociale de l'exil, le sens d'un déracinement plus sociologique que géographique a également été mentionné chez d'autres chercheurs. Utilisant l'expression d' " exil imaginaire ", Fouquet (2007) comprend le désir d'ailleurs et l'imaginaire migratoire de jeunes dakarois comme une forme d'exil, tandis que Noiriel (2006, cité par Fouquet, $2007: 83$ ) définit le déracinement " comme une perte, pour l'individu, des principaux repères et soutiens qui lui assuraient l'intégration dans un milieu ".

Si nous définissons l'expérience migratoire comme le rapport personnel et actif de l'individu avec l'évènement migratoire qui, elle, concerne tant la rencontre du sujet avec l'évènement (le vécu) que l'évènement lui-même (la migration $)^{7}$, la problématique exilique est alors quant à elle d'une tout autre nature : réflexive et relationnelle à la fois, universelle, subjective et singulière en même temps, sans cesse encadrée par de grandes tensions et ainsi hésitant entre le passif et l'actif, l'intérieur et l'extérieur, le positif et le négatif. En ce sens, l'exilé semble se rapprocher des personnages liminaires, au sens que donne Scarpa (2009), " figures bloquées sur les seuils, figées dans un entredeux constitutif et définitif " : " L'individu en position liminale [...] se trouve dans une situation d'entre-deux et c'est l'ambivalence qui le caractérise d'une

7 Nous éviterons néanmoins d'en délimiter des frontières temporelles, considérant qu'elles sont éminemment liées au contexte et à la subjectivité de l'individu, tout en nous interdisant cependant de les circonscrire à la seule expérience du voyage. 
certaine manière le mieux : il n'est définissable ni par son statut antérieur ni par le statut qui l'attend tout comme il prend déjà, à la fois, un peu des traits de chacun de ces états. [...] Dans la mesure où [le] personnage liminaire, faisant le détour par l'autre comme tout un chacun, ne parvient pas à revenir de cette altérité ; qu'il est, selon les circonstances et les contextes, un non-initié, un mal initié ou un sur-initié (voire le tout en même temps), il est placé souvent, dans le système des normes culturelles, du côté du pôle le moins positif ou le plus problématique " (Scarpa, $2009: 5-6)$. D'autre part, il n'y a pas un, mais des exils. Et enfin et surtout, exil et migration, ou expérience migratoire et exilique, n'ont pas un sens équivalent, de même qu'ils ne sont pas nécessairement dépendants I'un de l'autre. Ainsi, penser l'expérience exilique permet d'affiner la compréhension des expériences migratoires en approfondissant une de leurs dimensions. L'exploration de celle-ci incite à se pencher sur la littérature pour y déceler l'expression d'intériorités et d'intériorisations peut-être moins aisément lisibles dans les entretiens, ce qui expliquerait aussi en partie le délaissement de la notion par les sciences sociales.

\section{Expériences migratoires et exiliques : pour un renouvellement des sources et objets}

Nous ne souhaitons pas ici entrer dans les débats qui agitent les sciences sociales quant à la véracité des faits contenus dans la littérature, mais simplement, toujours sous le signe de l'expérience, souligner la porosité des textes littéraires et du récit de soi, porosité qui autorise à prendre pour source et objet d'étude des textes jusque-là majoritairement laissés par les sciences sociales à la critique littéraire.

D'aucuns, tels que Dorrit Cohn (1981), interprètent la littérature comme le seul médium permettant de dévoiler une part de l'intériorité de l'individu tandis que les relations sociales sont toujours confrontées à un certain degré d'incompréhension ou d'approximation. Sans adhérer entièrement à ces positions par certains côtés radicales, nous rejoignons plus volontiers les propos de Bonn (2000) qui soutient quant à lui que la littérature permet de dépasser les approximations de I'oralité et, par opposition à la littérature, de sa spontanéité : " le rôle de la littérature n'est-il pas de nous aider à nommer l'innommable, ou plus précisément ce qui jusque-là n'avait pas encore de nom dans cette accumulation de clichés qu'est en partie le langage commun : ce discours social qui nous permet de communiquer à partir d'un consensus d' "évidences", dont le but n'est pas tant d'être fidèles à ce qu'elles décrivent, mais de nous permettre de communiquer à son propos ?" (Bonn, 2000). L'ethnocritique de la littérature pose quant à elle "I'hypothèse d'une homologie possible, fonctionnelle et structurelle entre le rite et le récit littéraire " (Scarpa, $2009: 2$ ), fondée sur l'observation (concernant particulièrement le récit moderne) du "cadre ethnologique " sur lequel se meuvent les personnages. Le rite, au sein du roman, permet alors de mesurer en partie " le type de "socialisation" (en termes d'intégration, d'autonomisation, etc.) du personnage et sa plus ou moins grande réussite, son organisation formelle aussi peut servir à penser la narrativité " (Scarpa, 2009 : 4). Sans pour autant céder à une réduction de la réalité sociale au rite, nous suivons l'hypothèse posée par l'ethnocritique de la littérature selon laquelle les récits littéraires disent alors " quelque chose de la mise en marge [...] d'un ou de plusieurs personnages" (ibid.). 
Nous nous situons ici plus pleinement dans la perspective ricœurienne autour du concept $d^{\prime}$ " identité narrative ", Ricœur proposant des outils permettant de soulever les parallèles entre le récit de vie et le roman, telles que l'identité narrative et l'identité " mêmeté " ou "ipséité ". En effet, dans l'un comme dans l'autre, l'unité narrative d'une vie est objet de reconstruction : si nous ne sommes pas auteurs de notre vie comme on peut être celui d'un roman, le récit reste une tentative d'unification à postériori, une construction appuyée sur une forme de " mise en intrigue ". La performance narrative que sollicite l'enquêteur auprès de l'enquêté par exemple, ne saurait donc traduire la vérité d'une vie, mais seulement celle d'un instant et demeure ainsi éminemment liée à une situation, un contexte et des circonstances. En effet, n'importe quelle enquête fondée sur le récit de vie demande à l'enquêté un retour sur lui-même soustendu par un effort de cohérence (Boltanski, 2004), une " totalisation " de son chemin de vie, ne serait-ce même que pour se présenter, c'est-à-dire décliner son identité, résumer plus ou moins en détail ce que fut sa vie et ce qui le définit maintenant, en tant qu'individu. "Récit d'identité " (Anderson, cité par Candau, 1998 : 63), " discours de présentation de soi " (ibid.), " totalisation existentielle " (Candau, 1998) on retrouve ici la notion d'“ identité narrative " de Ricœur au sein d'un discours dont l'individu reste, plus ou moins consciemment, acteur " total " de sa construction. Bourdieu insiste quant à lui sur l'idée que l'enquêteur et l'enquêté partagent le même postulat (Bourdieu, 1986). Or, la linéarité à l'œuvre dans tout récit de soi est en partie historiquement liée à la production littéraire et à la littératie qui ont formaté une certaine manière de parler et notamment de soi (Bourdieu, 1986 ; Goody, 2007). En situation d'entretien, il reste implicite que cet effort de cohérence et de linéarité doit se faire : parfois les enquêtés s'excusent de "partir dans tous les sens " quand leur discours ne respecte pas de chronologie développant des relations claires de causalité. Mais la littérature, comme tous les arts, sait aussi s'affranchir de cette norme totalisante qui n'a rien de mimétique et reconnue comme historiquement et géographiquement construite (Bourdieu, 1986), rendant possible la restitution des différentes formes de fragmentation de l'expérience.

II semble alors a priori possible et heuristique de lire ces expériences migratoires et exiliques dans des textes littéraires quels qu'ils soient, ceux-ci ayant un statut épistémologique permettant d'approfondir la compréhension de ces expériences.

\section{Exils : des identités impossibles}

\section{"Première constriction " : exils congolais chez Biyaoula, entre retour et origines}

$L^{\prime} I m p a s s e^{8}$, le premier roman de Biyaoula nous permet dans un premier temps d'aborder une forme d'exil intérieur au Congo. Cet exil social y poursuit le narrateur depuis sa naissance et est réactivé dans le retour " au pays ", après la migration.

8 Le titre de cette partie a été choisi en référence à L'Impasse de Biyaoula dont la première des trois parties qui la composent est nommée " première constriction ". Celle-ci concerne exclusivement le retour du narrateur au Congo. 
À travers la logique de L'Impasse, Biyaoula déplace la focale avec laquelle est traditionnellement lue la place du migrant dans la société française - soit largement à travers des politiques d'intégration - pour lui donner une dimension à la fois transnationale et bilatérale. L'auteur souligne ainsi le continuum d'une " situation coloniale" (Balandier, 1951) s'exprimant à travers l'imaginaire collectif cristallisé autour de la migration. La confrontation de Joseph, le narrateur, avec son pays natal plus de quinze ans après son départ pour la France, va l'entraîner dans une forme de maïeutique dont il ne sortira pas indemne, le chemin vers la vérité se révélant une véritable "impasse ". Sous la plume de Biyaoula, les corps individuels, focalisant toute l'attention du narrateur, apparaissent peu à peu le miroir d'une violence symbolique bâtie de situations hégémoniques - au sens gramscien - et de dominations définissant les rapports entre l'Occident et I'Afrique dans le contexte de la mondialisation, et plus spécifiquement de ceux de la France et du Congo dans un contexte postcolonial. À l'instar de Black Bazar, l'extranéité des personnages s'établit ainsi comme le reposoir des diverses violences symboliques et autres assignations identitaires auxquelles ils sont soumis.

L'identité du narrateur de L'Impasse, marquée dès sa naissance, non pas finalement par une couleur de peau très sombre, mais plutôt par le regard qu'y posent les autres - et, très symboliquement, sa propre mère qui l'affuble elle-même du surnom de "Kala " ("le charbon ") - est en réalité irréconciliable. Dans le même temps que la couleur de sa peau le singularise et le marginalise, "ça ouvre en vous un précipice infini. Vous voyez tout à travers votre noirceur. Lugubre, que vous devenez. Vous n'avez plus goût à rien. Vous y construisez des labyrinthes dont vous ne parvenez plus à sortir. Où que vous allez, vous avez le sentiment de ne plus être à votre place "(Biyaoula, $1996: 18)$, elle lui confère un statut symbolique, archétypal. II est celui qui est différent et qui en même temps incarne une condition, celle de l'homme noir migrant dans un monde à la fois mondialisé et postcolonial. C'est cette vérité-là, immuable dans le temps du roman, que Joseph va finalement découvrir au fond d'une quête de lui-même impossible sortie de la Caverne platonicienne - pour comprendre qui il est dans le monde dans lequel il vit. Plus que le portrait de Joseph, le roman dresse celui des relations compliquées entre le Congo et la France incarnées paradigmatiquement par le personnage du migrant, qui est alors celui qui, d'un côté, ouvre les yeux sur ces mondes sociaux et, d'un autre, est lui-même implacablement déterminé par ces derniers.

Néanmoins, si la quête identitaire du personnage principal de L'Impasse apparaît très tôt vaine et sans autre solution que la mort, la folie ou le renoncement à soi, la migration en elle-même est positivement représentée pour l'ouverture d'esprit et le recul sur son pays d'origine qu'elle donne à Joseph. Le départ du narrateur vers l'Occident se dévoile en effet rétrospectivement comme une possibilité d'éloignement d'avec l'assignation que son entourage fait constamment peser sur lui à travers son surnom. En outre, c'est dans le retour au pays, en étant sans cesse surpris et touché par les stratégies de dissimulation de la part de son entourage de toute extranéité de leur condition d'hommes et de femmes noir(e)s, africain(e)s, congolais(es), que Joseph commence à ouvrir les yeux et à se poser des questions demeurées jusqu'alors inaccessibles, regardant ce dont il s'était tenu éloigné depuis longtemps. La migration, c'est-à-dire ici, le lieu de rencontre entre l'imaginaire de la migration et de l'Occident, et spéciale- 
ment Paris et la France, joue ici le rôle de révélateur d'un état social de la société congolaise. L'expérience migratoire de Joseph lui permet d'y porter un regard différent, parce qu'enrichi de l'expérience de la confrontation avec le mythe français et parisien.

Faisant figure de catalyseur, le travestissement des corps congolais (décoloration de la peau vers une teinte plus claire, teinte et lissage des cheveux, etc.) visant à tendre vers le modèle occidental ne cesse de perturber Joseph, qui se demande sans cesse "pourquoi ? ". En commençant à se poser des questions et à s'indigner, il entre dans un jeu dangereux. Joseph se met en quête de chercher cette inconnue, ce " $x$ " (Biyaoula, $1996: 86$ ), qui a conduit sa mère, ses sœurs et toutes ses connaissances féminines à se décolorer les cheveux et la peau, et les hommes, à les inciter à le faire. Le corps individuel se révèle comme le lieu où s'affichent tous les efforts fournis pour s'extraire d'une condition noire vécue comme honteuse et à dissimuler avec force d'artifices. À travers la multitude de ces visages "maquillés ", décrits par le narrateur comme victimes de mutilations, c'est l'ensemble du corps social qui apparaît mutilé, meurtri dans son identité et dissimulé sous une multitude de faux-semblants. La violence faite aux corps est ainsi appuyée par le tranchant de la plume de l'auteur :

"Elle [Mère] a toujours son allure de phoque, mais plus son teint d'antan qui faisait sa fierté. Faut comprendre que mère faisait partie de l'avant-garde en matière de "maquillage" qui dans le français de chez nous signifie décoloration de la peau. Ah ! Elle ne doit plus faire ostentation de son teint de Blanc, comme elle aimait dire ! C'est un visage d'Arlequin tout recouvert de boutons et de squames qu'elle trimballe. Peut-être il est à peine moins noir que le mien " (Biyaoula, $1996: 37$ ).

D'autre part et si, au début du roman, le retour de Joseph au Congo après quinze ans d'absence semble pouvoir contenir les germes d'une nécessaire réconciliation avec lui-même, L'Impasse ne lui laisse pas cette chance, emmuré qu'il est dans une identité intrinsèquement problématique. Sa présence en France avant son retour apparaît en effet marquée par un déni de lui-même dans ce qu'il fut au Congo, de même que par " une espèce d'indifférence " (Biyaoula, 1996 : 18) qu'il porte à sa famille congolaise tandis qu'il est en France, principalement par peur des confrontations - avec sa famille, mais aussi avec lui-même induites par le retour. Notamment, parce que ce retour (r)établit Joseph entre deux identités que la société congolaise rapporte à la marge : I'une est celle de "Kala ", déjà exclu, moqué, dès sa naissance marginalisé par sa couleur de peau. L'autre est dans le retour même, qui installe Joseph dans un personnage liminaire (Scarpa, 2009), passant à côté des rites de passage qui font du migrant de retour un migrant accompli l'empêchant de sombrer dans la masse de ceux relégués dans la honte de l'absence de réussite conférée par la migration?. Plus que tout, c'est ce rôle, imposé par sa famille qui atteint Joseph dans son identité profonde - rôle qui s'inscrit dans cette forme de ritualisation du retour à laquelle il est attendu que le migrant se plie, faisant à la fois I'honneur de sa famille et prouvant le bien-fondé et la réussite de son départ en migration. Ainsi, lorsqu'il arrive habillé de manière simple - ce qui est contre l'attente des siens l'accueil familial à l'aéroport se fait crispé. De même, l'épisode douloureux (pour

9 Pour plus de précisions sur la ritualisation du retour du migrant et le nécessaire positionnement discursif du migrant de retour, voir Pénicaud (2017). 
ne citer qu'un seul exemple supplémentaire) pendant lequel le frère aîné de Joseph, Samuel, prétextant une course avant de rentrer à la maison familiale, le conduit directement de l'aéroport acheter au magasin brazzavillois " Les habits de Paris " un costume qu'il est sommé d'enfiler avant de rejoindre la famille et prétendre qu'il s'est changé avec ses propres affaires restées dans sa valise. Joseph proteste vivement, mais fini par se soumettre à son aîné :

" II [Samuel] s'éclaircit la gorge puis il me dit qu'il y a des règles à respecter que je ne les aime peut-être pas, les costumes, mais que je suis un Parisien ${ }^{10}$, que le Parisien a une image à défendre, que pour eux, les gens de ma famille, ce sera la honte insoluble qu'il y ait parmi eux un Parisien qui ne ressemble pas à un Parisien, qu'il faut que je pense aux miens, que je l'ai peut-être remarqué à l'aéroport combien ils ont été peu enthousiastes en voyant comme je suis alors qu'autour de nous les autres Parisiens faisaient honneur au pays d'où ils venaient ainsi qu'à leur famille, que c'est une question de prestige, que c'est important le prestige [...] Je réponds à mon frère qu'il n'ignore pas que je vis à Poury [...] Samuel s'énerve un peu. II me rétorque, que je le veuille ou non, que pour les gens je suis parisien puisque je viens de France » (Biyaoula, $1996: 39-40$ ).

Comme nous y invite l'ethnocritique de la littérature, il est possible de traduire du positionnement de Joseph par rapport à une forme de ritualisation du retour son propre degré de socialisation dans une société qui fut la sienne, mais qui l'accueille ici en tant que migrant de retour. Dérogeant à ce qui est attendu de lui, il est mis à la marge, par la sphère qui pourrait pourtant être la plus intégratrice dans ce retour : celle de la famille. Ainsi, Joseph, doit, contre sa volonté, endosser le costume - au propre comme au figuré - attendu de celui qui a migré en France et rentre au Congo " pour les vacances ". II fallait donc que Joseph retourne au Congo pour qu'il comprenne qu'il est " parisien " : "Tu es parisien, toi ! [...] II faut que tu comprennes que tu es parisien Joseph ! " (Biyaoula, 1996 : 94). À cette assignation à prétendre être ce qu'il n'est pas, à le " comprendre " et à y croire, Joseph réagit par de violentes céphalées dont la nature itérative annonce l'insolubilité de sa condition. La fracture identitaire qui ne cessera de fissurer son " moi " profond le perd : "Des minutes entières que je tente vainement d'y apporter des réponses, de savoir si j'existe, si je peux exister à Brazza, si notre lot à nous ce n'est pas seulement d'être... " (Biyaoula, 1996 : 132). Le retour dans sa famille congolaise s'accompagne en effet de l'obligation à renier tout ce qui le définit : ses choix, ses caractéristiques physiques, ses vêtements, sa sexualité, son métier, ses convictions religieuses, etc. Cet ensemble de sommations, auquel Joseph ne peut résister s'il ne veut pas rompre définitivement avec son passé et ses attaches congolaises, le conduit à souffrir d'une forme de désindividuation.

C'est ainsi que la première partie de L'Impasse, celle du retour au Congo nommée "première constriction ", se clôt sur les pensées de Joseph : "Je me sens ignoble. Je me demande si je me regarderais encore dans une glace. Je voudrais être ailleurs ou crever... [...] J'ai besoin d'aller faire un tour tant je suis au désespoir, tant je me sens sale, avili, éreinté jusqu'au bout de l'âme " (Biyaoula, 1996 : 141). La cassure est en effet irréparable et le retour de Joseph en

10 Le titre de "Parisien " est donné, dans le milieu de la Sape (Société des Ambianceurs et des Personnes Élégantes) à celui qui, de Paris, revient à Brazzaville (pour plus de précisions voir Gandoulou, 1984). 
France ne recollera pas cette identité diffractée. Bien au contraire. Une multitude de questions ne cessent de l'assaillir et toutes gravitent autour d'un même paradigme : "Nous autres [Congolais], on ne laisse pas de jouer à cache-cache avec nous-mêmes, de croire qu'on est libres dans notre tête, que tout ce que I'on fait, c'est par rapport à nous, alors qu'on passe notre temps à nous faire des cheveux sur l'image qu'ils ont de nous, ceux d'en face [Français, Occidentaux], à vouloir entrer dans leurs bonnes grâces, à les pasticher, à s'échiner à leur ressembler, à leur démontrer qu'on est tous bien " (Biyaoula, 1996 : 183). L'idée de la persistance de la supériorité de l'homme blanc sur l'homme noir le ronge, l'accapare.

Cet aspect se retrouve chez Mabanckou dans la dénonciation d'une forme de désolation (Arendt, 2005), d'exil de la culture et des valeurs de la société d'origine dont furent responsables la colonisation et son travail hégémonique, se traduisant notamment par la perte du sens des liens. Le père du narrateur de Black Bazar permet à l'auteur d'aborder cette question : "Son travail de boy chez les Européens du centre-ville était comme une vengeance, une occasion de prouver aux vieux du quartier qu'il avait réussi sa vie. D'ailleurs, ces vieux, il les qualifiait tous $d^{\prime \prime}$ australopithèques" parce qu'il estimait qu'ils étaient fermés d'esprit, sans culture, sans vision sur les grands problèmes du monde " (Mabanckou, 2009 : 198).

Quant au narrateur de L'Impasse, celui-ci voit, lors de son retour en France, se renforcer son irritabilité face à la condition des migrants et aux actes de racisme à son égard. La " deuxième constriction " de Joseph se rapporte à ce retour-là, en France, que l'on peut aisément rapprocher de l'expérience de la France des personnages de Mabanckou et N'Sonde qui eux, ne mettent pas en scène le retour ou la vie au Congo - ou du moins pas dans la même mesure.

\section{Des exils de France : Mabanckou et N'Sonde}

En France, l'exil prend des formes différentes chez ces auteurs, mais toutes tendent vers une nature sociale. Chez Mabanckou comme chez N'Sonde, I'exil intérieur dans le pays dit parfois " d'arrivée " prend la forme d'une impossibilité à être soi indépendamment de ce à quoi les personnages sont sans cesse rapportés, c'est-à-dire leurs origines, leur couleur de peau, les banlieues où ils vivent. Aussi, dans Black Bazar, les identités sont-elles directement déclinées dans les noms des personnages : "Roger le Franco-Ivoirien ", "Paul du grand Congo ", "Olivier du petit Congo ", "Vladimir le Camerounais ", "Yves I'Ivoirien tout court ", "Pierrot le Blanc du petit Congo ", etc. De même, les personnages sont parfois nommés par leur carnation : le personnage principal de Black Bazar tombe ainsi amoureux d'une fille nommée "couleur d'origine " en raison de l'aspect très foncé de sa peau. Si Biyaoula soulignait déjà l'idée que " au pays, on n'aime pas trop une couleur pareille " (Mabanckou, 2009 : 69) par ailleurs reprise par N'Sonde dans Le Silence des esprits ${ }^{11}$, Mabanckou met directement en relief les relents évolutionnistes intégrés à la logique migrante congolaise qu'il a choisi de dépeindre - à savoir que le migrant venu en France acquiert un statut de supériorité par rapport à " celui qui est resté "- dont les person-

11 Dans Le Silence des esprits, le petit garçon qu'était Clovis est lui aussi stigmatisé en raison de la couleur de sa peau jugée trop sombre. 
nages de cet auteur, et notamment les personnages féminins, font les frais. Par exemple, la violence avec laquelle est décrite Rose, récemment arrivée du Congo, lorsqu'elle se met à danser et dont la gestuelle est alors qualifiée de " préhistorique " (Mabanckou, 2009: 170) : " on aurait dit un être qui, au lieu de descendre du singe comme tout le monde, y retournait irrémédiablement " (ibid.).

Les romans de N'Sonde quant à eux, traduisent bien, un peu chacun à leur manière, le sentiment de déracinement - (" ne pas avoir de place reconnue et garantie par les autres ", d'après Arendt) - des migrants ou des " deuxièmes générations". Ainsi, si Le Cœur des enfants léopards s'ouvre avec le monologue du narrateur qui se trouve dans une cellule de garde à vue, entrecoupé des violences de policiers, il est d'abord le récit de l'enfance et de l'adolescence de trois jeunes amis d'origines différentes qui grandissent dans un quartier de la banlieue parisienne. Par la couleur de leur peau et le quartier où ils habitent, le narrateur et son ami Drissa regardent impuissants les assignations et l'exclusion se lever et s'accroître en même temps qu'ils grandissent : face à ces difficultés et tandis que le narrateur tente de rester dans le droit chemin, Drissa sombre, encore adolescent, dans la délinquance et la folie. Mireille, le troisième élément du trio et amante du narrateur, va d'abord se détacher de Drissa puis s'éloigner petit à petit du narrateur jusqu'à le quitter. Elle poursuivra ses études, coupera les liens qui I'unissaient au quartier et se constituera un capital symbolique plus important. La rupture sentimentale d'avec Mireille marque également pour le narrateur la rupture entre la possibilité d'une vie en accord avec ce qu'il est et le milieu dont il est issu (lieu et amis) et la trajectoire prise par Mireille. Mireille sort de la cité, mais, contrairement à Drissa et au narrateur, elle est " blanche " de peau. Le narrateur, ivre le soir de sa rupture avec Mireille, se dispute avec deux policiers et frappe l'un d'eux mortellement. C'est la raison pour laquelle il se retrouve en garde à vue dès le début du roman et subit la haine d'un de ses gardiens.

Les personnages du Silence des esprits, Clovis Nzila et Christelle incarnent tous les deux à leur manière une forme d'exil et d'errance différente. C'est dans les formes de communication qu'ils vont mettre en place entre eux, parfois difficilement, qu'émergera la possibilité d'une réconciliation avec eux-mêmes et celle d'un pardon pour Clovis : "Deux errances encore hésitantes s'embrassaient à tâtons " (N'Sonde, 2010 : 17-18). Pour Christelle, c'est celle de la solitude dans un monde désindividualisé et pour Clovis, c'est l'errance en France d'un ancien milicien congolais sans papiers et sans logement, qui semble à Christelle perdu sur le pont d'Austerlitz : "Christelle m'identifia comme un homme seul au milieu de rien, recroquevillé dans sa peau, avec la tête qui aurait préféré disparaître entre les épaules " (N'Sonde, 2010 : 20). Lui, "illégal [...] n'avai[t] ni ami ni compatriote " (N'Sonde, $2010: 25$ ). Les lieux de leur rencontre, la gare puis le train, sont autant de lieux de passage, de seuils, soulignant leur placement commun sous le signe de la solitude et de la désindividuation. Par exemple, le narrateur ne prend un nom qu'à la page 40, après que Christelle l'ait sauvé dans le train d'un contrôle d'identité et lui ait " tend[u] la main pour [le] saluer " (N'Sonde, $2010: 40$ ), retrouvant par ce geste d'humanité, une identité. Clovis devient, à cette page 40 , un individu à part entière, lui qui n'était sur le pont d'Austerlitz " qu'un triste reflet d'humanité " (ibid. : 23). 
Enfin, I'exil prend une autre forme encore sous la plume de Mabanckou dans Black Bazar, celle du spectre d'une injonction identitaire pour l'écrivain qu'est le personnage principal :

" Je me suis demandé pourquoi les Haïtiens sont soit écrivains de génie soit chauffeur de taxi à vie à New York ou à Miami. Et quand ils sont écrivains, ils sont en exil. Est-ce qu'on écrivain doit toujours vivre dans un autre pays, et de préférence être contraint d'y vivre pour avoir des choses à écrire et permettre aux autres d'analyser l'influence de l'exil dans son écriture ? [...] Louis-Philippe me dit que sans le mal du pays rien ne sort même si on voit des oiseaux qui s'agitent dans les arbres. Or moi aussi je suis loin de mon pays, je me sens en exil, est-ce que je vais passer ma vie à pleurer sur ça ? "

(Mabanckou, 2009 : 181-182).

L'auteur s'interroge : comment être auteur en étant d'origine étrangère ? Lorsque l'on vient d'ailleurs, peut-on encore dire autre chose que l'exil ? Soulignant en outre les poncifs liés à la distinction entre " civilisations de l'écriture " et "civilisations orales ", la réduction à l'oralité et la dénégation de pleine historicité des sociétés africaines, ce passage résume assez bien le double paradoxe identitaire dans lequel se situe l'écrivain noir africain :

"Écoute, mon gars, sois réaliste ! Laisse tomber tes histoires de t'asseoir et d'écrire tous les jours, y a des gens plus callés pour ça, et ces gens-là on les voit à la télé, ils parlent bien, et quand ils parlent, y a un sujet, un verbe, et y a un complément. Ils sont nés pour ça, ils ont été élevés dans ça, alors que nous autres, les nègres, c'est pas notre dada, l'écriture. Nous c'est l'oralité des ancêtres, nous c'est les contes de la brousse et de la forêt, les aventures de Leuk-le-Lièvre qu'on raconte aux enfants autour d'un feu qui crépite au rythme du tam-tam. Notre problème c'est qu'on n'a pas inventé l'imprimerie

et le Bic, et qu'on sera toujours les derniers assis au fond de la classe à s'imaginer qu'on pourrait écrire l'histoire du continent noir avec nos sagaies. Est-ce que tu me comprends ? En plus, on a un accent bizarre, ça se lit aussi dans ce que nous écrivons, or les gens n'aiment pas ça. D'ailleurs, il faut avoir un vécu pour écrire. Et toi, qu'est-ce que tu as comme vécu, hein ? Rien ! Zéro ! Moi par contre j'aurais des choses à raconter parce que moi je suis un métis, je suis plus clair que toi, c'est un avantage important "

(Mabanckou, 2009 : 13-14).

Enfin, le narrateur de Black Bazar dont la nostalgie se heurte à I'hostilité raciste de son voisin antillais nous permet de rappeler que ce sentiment, corollaire de l'exil, procède également du social, bien que la doxa le réduise plus facilement à un enracinement relatif à la métaphore végétale : "II [le voisin] prétend qu'il y a des bruits et des odeurs quand mes amis et moi nous préparons de la nourriture et écoutons de la musique de notre pays d'origine pour oublier un peu les tracas de la vie quotidienne. La nostalgie, il ne sait pas ce que c'est. Lui, son pays c'est la France, et il me gueule sa fierté d'être né français de souche " (Mabanckou, 2009 : 36). Barbara Cassin le souligne bien, la nostalgie procède de I'enracinement, mais au sens weilien : " Enracinement et déracinement : voilà la nostalgie "(Cassin, 2013 : 20). Alors la nostalgie est aussi un objet social. En effet, précise la philosophe analysant sa propre nostalgie de la Corse dans laquelle elle $n^{\prime}$ a aucune origine familiale, $n^{\prime} y$ est pas née et $n^{\prime} y$ a pas vécu dans sa jeunesse : "C'est seulement parce que j'y suis hospitée que je m'y sens chez moi. D'autres y ont des racines, plus de racines, et ils m'accueillent " (Cassin, 2013 : 14). À la question "Quand donc est-on chez soi ? " (Cassin, 2013 : 132), la 
philosophe répond, s'alignant sur les positions d'Arendt et de Weil : "Quand on est accueilli, soi-même, ses proches et sa, ses langues " (ibid.).

\section{L'incommunicabilité des exils. De l'écriture à l'errance, la mort ou la folie}

Dans Black Bazar, les questionnements du personnage principal relatifs aux multiples exils de l'Africain en France sont en quelque sorte recyclés pour devenir des mots sur du papier : c'est en devenant écrivain que le narrateur parvient à boucler la boucle du roman - le lecteur apprend à la fin de l'ouvrage que le narrateur en est aussi l'auteur et écrit le livre au fur et à mesure que le lecteur le lit. Néanmoins, le destin de tous les personnages du corpus ne converge pas vers cette heureuse résolution. Chez N'Sonde comme chez Biyaoula, les romans se terminent par la mort, la folie ou l'errance. Ce que nous souhaiterions ici souligner, c'est le dénominateur commun à ces trajectoires descendantes, soit l'incommunicabilité des conditions des personnages et par là, l'impossibilité même, pour eux, de faire société. La possibilité de faire société passe, selon Weil, par un nécessaire " enracinement " social ou, selon Arendt, par la reconnaissance (et la garantie) d'une place en ce monde, toutefois, les auteurs du corpus nous soulignent d'abord qu'elle procède de la communication entre les individus. Lorsque celle-ci est rompue entre l'individu et la société, que celui-ci est marginalisé, même discursivement, quand l'espace d'une parole, d'un échange, n'est plus possible, alors la sociabilité est en péril (Katambwe, 2011). En effet, comme Bonn (2000) le souligne à propos des écritures maghrébines, pour ces auteurs qui disent l'exil de l'intérieur, ces écritures " aphasiques " répondent au besoin de " mettre en mots un vécu stupéfiant de cette communauté, afin de permettre à [leurs] lecteurs d'en comprendre l'inédit " (ibid.). Nous nous alignons ici directement sur les analyses de Bonn indiquant que ces écrivains doivent en quelque sorte trouver les mots pour dire "un vécu aphasique "(ibid.) de la migration, accouchant alors nécessairement d'une nouvelle créativité littéraire : " c'est précisément lorsqu'il est sommé ainsi de trouver des mots nouveaux pour des vécus aphasiques, que l'écrivain va dans un premier temps décevoir I'attente par trop balisée à laquelle il doit répondre. Les grands textes qu'on vient de citer ne sont pas les descriptions du quotidien de l'immigré que les lecteurs attendent alors. D'emblée, ces écrivains déjà confirmés vont inventer un dire, plus : une poétique nouvelle pour cet espace de stupeur qu'est l'expérience de l'émigré, car elle n'a jamais été dite jusque-là de l'intérieur " (ibid.). Dans un geste parallèle à celui de Bonn, cette partie développe la double dynamique de marginalisation inhérente à ces écritures d'un vécu lui-même marginalisé, non seulement dans l'espace social, mais aussi et par là, dans et par le discours " une marginalisation certaine par rapport à une attente stéréotypée (attente contradictoire d'un discours prévisible sur une réalité inédite), mais aussi un dire de la stupeur productif dans sa marginalité même " (ibid.) - : dans le cas de Biyaoula et de N'Sonde, la mort, sociale ou non, la folie ou l'errance en dessinent les contours tandis que s'annulent brusquement les discours des personnages concernés, comme dans le cas du Silence des esprits, ou que ceux-ci s'orientent vers des monologues autocentrés ( $\left.L^{\prime} I m p a s s e\right)$ ou encore fermés à tout récepteur du monde visible (Le Cour des enfants léopards). 
Chez Biyaoula, la troisième et dernière partie de l'ouvrage, "La mue ", consacre l'effondrement de Joseph qui n'a alors le choix qu'entre la folie ou la mort, annoncées par des céphalées et conséquences de l'incommunicabilité de sa condition et de l'illusion du mensonge. La voie qu'il choisira est celle que lui montre son psychiatre, le Docteur Malfoi, dont le nom est sans équivoque : celle de la reddition, de l'illusion et du mensonge. Avec le Docteur Malfoi, Joseph se " rend " et la vie devient plus simple. Au début de sa cure, Joseph explique :

"Moi je l'admire de plus en plus le Docteur Malfoi. Sûr que sans lui, je serais encore

là à me torturer, à me déchirer inutilement, à m'exciter les neurones, à me rendre malade pour tout, à me poser des tonnes de questions, à éviter de me regarder dans un miroir. C'est vrai qu'il m'a ouvert les yeux le gentil docteur ! Grâce à la petite amnésie qu'il a installée dans ma tête, je ne le trouve ni beau, ni laid mon visage tout noir, à améliorer peut-être au niveau de la couleur, mais c'est tout " (Biyaoula, 1996 : 266).

C'est alors le début d'une rapide transformation pour Joseph qui se met à se décolorer les cheveux, s'achète de nombreux costumes, prend trente kilogrammes et se réjouit à l'idée du succès qu'il rencontrera à Poury (sa résidence en France) et à Brazzaville. II change de fréquentations et de lexique pour qualifier ceux qu'il voyait jusque-là d'un œil critique, empruntant des qualificatifs tous plus laudatifs les uns que les autres. Les lieux qu'il fréquente ne sont eux aussi plus les mêmes. II se met à boire. Et surtout, il change de nom, se faisant désormais appeler non plus Joseph, mais Jo. La mue est complète. II ne s'interroge désormais que pour se regarder : "De plus en plus, je me demande vraiment comment toutes ces années j'ai pu passer à côté de choses si revalorisantes ! "(Biyaoula, $1996:$ 271). La " guérison " de Joseph se fait progressivement, au rythme de ses visites chez le Docteur Malfoi, "spécialisé en psychologie africaine " :

"Ah, c'est le grand hourra, le bonheur !... De plus, cette impression d'avoir un gros trou en moi [ressentie au début de sa "guérison"] : quasiment disparue ! Comme si mon gras le comblait! Alors je m'empresse d'aller acheter des costumes, des chemises, des cravates et des chaussures " (Biyaoula, $1996: 274)$.

" Jo " s'est transformé à la fois physiquement et intellectuellement : il a perdu tout jugement critique et se nourrit d'illusions. Entre temps, sa compagne I'a quitté, enceinte d'un fils qui figure pour Joseph un futur avorté, lui qui ne connaîtra jamais son enfant. Lors d'une soirée à laquelle Joseph aurait détesté se rendre, "Jo " rencontre Justin et se trouve soulagé de trouver enfin plus noir que lui. Justin, que tout le monde appelait "Le Goudron " lui raconte son enfance malheureuse qui ressemble étrangement à celle de Joseph. Le lendemain, Joseph apprend que Justin est mort dans la nuit, ayant écrasé sa voiture au fond d'une impasse. Avec Justin, c'est la mort symbolique de Joseph, pris dans l'impasse d'une condition impossible. Quant à Jo, il envisage dès le lendemain de demander au Docteur Malfoi " s'il ne connait pas une méthode plus rapide, plus efficace, plus radicale pour m'effacer la mémoire et me donner de bons souvenirs... " (Biyaoula, 1996 : 327). 
C'est le même non-choix de la folie que réalise le narrateur du Cœur des enfants léopards, bien que le contexte et les causes n'en soient apparemment pas les mêmes. Dès le début du roman, le narrateur du Cour des enfants léopards est en garde à vue et le lecteur comprend petit à petit les raisons qui I'ont amené à choisir une forme de folie (à l'image de son ami Drissa), plutôt que d'écouter une justice en laquelle il n'a pas confiance, ayant trop souvent été victime d'injustice ou de racisme. Cette situation l'amène en réalité à stopper toute communication avec le monde social pour trouver refuge dans celui des ancêtres. Lui, dont la somme des assignations identitaires a conduit à brouiller le rapport au monde, se replace par rapport à ses geôliers dans une filiation (masculine) qui leur est à la fois inaccessible et incompréhensible. La généalogie de ses ancêtres lui permet de replacer son identité et de se situer dans une collectivité : celle des "enfants léopards "12. Comme chez Joseph dans L'Impasse, le sentiment d'injustice et la fracture d'une identité inconsolable amènent le narrateur et Drissa à se poser " trop de questions " et l'entraînent vers le mutisme et la folie. Une " folie " incomprise par les psychiatres - trahissant ce que le narrateur ressent à la fois comme une incompréhension de la part d'un Occident très rationnel :

"Professeur, docteur en psychiatrie ou spécialiste de je-ne-sais-quoi, assis confortablement sur tes livres, entends-tu les voix dans ma tête ? [...] Essaie une fois [...] de communiquer avec les esprits qu'invoque l'ancêtre, ils te parleront de moi et des léopards, ils t'enseigneront notre vie [...] abandonne l'idée de soigner mes maux de léopard, c'est mon unique jardin, ma seule richesse " (N'Sonde, 2007 : 38-39).

L'ouvrage de N'Sonde souligne différentes réalités de l'exil. II y a d'abord celui des parents pieds-noirs de Mireille ; il y a ensuite l'exil intérieur vécu dans le quartier, qui se rapproche assez de la désolation arendtienne; celui que ressent à l'école le tout jeune narrateur juste arrivé du Congo et que Mireille, sans doute, parce qu'elle est elle-même fille d'un exil que sa mère porte constamment en elle, est capable de comprendre : " fantastique Mireille qui compris instinctivement la blessure de l'exil, nous n'avions que cinq ans !" (N'Sonde, $2007: 82$ ), qui est ici un exil transmissible, à la fois de déracinement géographique, mais proche également de l'exil arendtien et weilien chez cet enfant qui a perdu ses repères ; et enfin, l'exil salvateur, celui de la folie, celui d'une filiation retrouvée et d'une identité réconciliée dans une communauté imaginée. C'est aussi celui d'un renoncement au monde. Dans une position très proche du personnage de Joseph à la fin de L'Impasse, le narrateur s'adresse ainsi au psychiatre : "Offre-moi un doux exil. Je veux la paix des déments" (N'Sonde, 2007 : 40). Comme dans L'Impasse, c'est l'incommunicabilité de l'exil qui amène le narrateur à se retourner vers ses ancêtres, communauté à la fois imaginée et le replaçant dans une identité, une filiation qui lui était jusque-là déniée. Puisant sa force dans le secours des ancêtres, le narrateur brandit alors, dans une posture de repli, la revendication d'une identité dans l'ailleurs, puisqu'ici, il ne se trouve pas "sa place " : "Capitaine, j'suis comme Drissa,

12 Chez les Kongos, le léopard, le ngo, incarne la force (MacGaffey, 2000). Associé à la puissance des chefs, eux-mêmes considérés comme des léopards, il est celui qui possède l'autorité et le pouvoir, notamment de vie et de mort. L'une des explications de l'origine du mot Kongo l'attribue directement au ngo, en faisant le "pays des léopards" (ko-ngo) (Soret, 2005). 
j'suis parti, t'as plus que mon corps, cette carcasse de doute et de complexes [...] moi je pars, ciao ! " (N'Sonde, $2007: 133$ ). Ce positionnement ne peut nous empêcher de penser à la posture de " repli » décrite par Burgos (1982) et inspirée des travaux sur l'imaginaire de Gilbert Durand, marquée par l'aménagement de " refuges, de lieux clos » (Burgos, $1982: 127)$.

Dans Le silence des esprits, l'absence de communication et de communion avec le monde extérieur se traduit d'abord par le sentiment de solitude de Clovis. Ce sentiment est mis en parallèle avec celui de Christelle, aux traits " mélancoliques "(N'Sonde, $2010: 28$ ), perdue dans une foule anonyme et dans un monde qu'elle peine à déchiffrer, n'en ayant pas toutes les clés. Chez Christelle, le lexique de la domination et de l'asservissement au travail (un travail d'aidesoignante qui lui plaît par ailleurs et dans lequel elle s'oublie et qui lui donne l'impression d'être utile) passe notamment par le langage. Christelle est celle qui préfère souvent se taire, aspect révélateur de sa condition de dominée : "Pour dire ses joies et ses peines, Christelle ne possédait qu'un registre de maigres mots ! " (N'Sonde, 2010 : 44). Clovis, lui, ne tend qu'à pouvoir rebondir une fois en France, considérant la migration comme la possibilité d'une rupture et du début d'une possible résilience, il demeure cependant dans une impasse " impossible de tourner le dos à mon passé, d'effectuer la grande rupture pour m'en aller vers l'ailleurs, l'espoir d'une vie d'étoiles, le bonheur " (N'Sonde, 2010 : 51) -, que seule Christelle semble pouvoir ouvrir par la libération d'une parole rendue possible par la confiance qui s'est établie entre eux : "Parle, ça te fera du bien!" (N'Sonde, $2010: 63$ ).

La difficulté de l'émergence du récit du parcours de Clovis encouragé par Christelle trouve un commencement dans l'enchâssement du récit moins personnel du mythe des origines, au " grand conseil de Nzambi A Mpoungou, créatrice de tout ce qui se voit ou ne se voit pas " (N'Sonde, 2010 : 54). La mise à distance permise par celui-ci permet à Clovis de se dévoiler peu à peu en amenant Christelle à parler un même langage et ainsi à pouvoir déchiffrer son histoire. Il résout ainsi en quelque sorte le problème face auquel se trouve le narrateur du Cour des enfants léopards qui lui, ne sachant trouver aucune oreille attentive, s'enfonce dans un mutisme insoluble : se retournant vers ses ancêtres, il fait de cette référence une force lui permettant de fuir, de s'aménager un espace imaginé en mettant à distance une réalité où il ne trouve pas sa place. Quant à Clovis, le recours à la mythologie lui permet, d'une part, de raconter en mettant à distance l'horreur de la guerre et, d'autre part, de donner un sens à ce qui n'en a pas (exactions, violences, etc.).

Pour se raconter (ou d'abord, éviter de se raconter) et à l'instar du personnage du Coeur des enfants léopards, Clovis va faire émerger le récit en se situant dans une filiation - de sa mère, de sa grand-mère - placée sous le signe de la colonisation, de ses conséquences et d'une forme d'exil, fille de ces dernières. Comme le narrateur de L'Impasse, Clovis est doté d'une peau très sombre qui le met, dès sa venue en un monde marqué de rapports raciaux, en posture d'être rejeté et mal-aimé. II faudra attendre la page 136, les pleurs et les supplications de Christelle, pour que Clovis fasse enfin le récit de sa propre histoire et que I'on apprenne qu'il fut, après la séparation d'avec sa sœur jumelle, enfant des rues 
à Brazzaville ${ }^{13}$. Sans repère, il est l'adolescent livré à lui-même d'une société en mal de transmission et de continuité, incarnant, d'une part, le clivage entre générations au sein de la société congolaise " ma génération " et, d'autre part, entre les différentes classes sociales (ceux qui ont des diplômes, de l'argent, des enfants en Europe) : " Je m'en allais pieds nus dans la poussière, sous la chaleur ou la pluie, observant avec envie la jeunesse dorée, heureuse et bien nourrie. Les Mercedes climatisées, costumes trois-pièces, ventres bedonnants, deux femmes, quatre maîtresses et les enfants légitimes en Île-de-France. " (N'Sonde, 2010 : 146). Situation qui le conduit à être facilement manipulable et aisément mis au service de la violence. Le récit de Clovis, en forme de réconciliation salvatrice d'avec lui-même, prend fin avec le pardon de Christelle qui a su écouter et comprendre son histoire, lui offrant enfin une place au sein des autres, une humanité en ce monde.

Néanmoins, si l'émergence de son récit eut pu présager une conclusion positive et le début d'une résilience pour Clovis, le roman ne lui laisse pas cette chance et se termine dans une (autre) impasse aux couleurs de l'errance. Clovis est arrêté dans la rue par la police française le lendemain de son récit et Christelle n'en saura jamais rien. Les "forces de l'ordre " incarnent ici le refus de faire société avec les migrants et le récit salutaire des multiples exils de Clovis en est en quelque sorte annulé : "Mon envol d'un instant finit par se briser, anéanti au contact de l'acier et de la loi ! Je continue mon errance vers nulle part, parqué comme une bête, l'amertume au cœur et les rêves en lambeaux " (N'Sonde, $2010: 171$ ). La migration constitue ici un leurre pour la résolution d'un exil antérieur, venant, de par les conditions d'accueil rencontrées, empiler de multiples expériences exiliques. L'errance n'apparaît que comme la somme de ces derniers. N'Sonde met en lumière à la fois la diversité et l'universalité de l'expérience de l'exil. Rejetant, comme dans Le Cour des enfants léopards, toute forme d'exotisme intrinsèque à celui-ci, ces récits témoignent de la possibilité de sa non-concomitance avec l'expérience de la migration. D'ailleurs, l'exil ne naîtra pas de la rencontre avec une altérité géographique, sociale, etc., expérience que pourrait incarner la migration, mais au contraire, s'apaisera dans la rencontre avec celle-ci, incarnée par Christelle qui partage avec le narrateur une même exiliance (Nouss, 2015), dans le dialogue et le pardon.

\section{Conclusion}

Ainsi, chez Biyaoula, comme chez Mabanckou ou N'Sonde, l'expérience migratoire est utilisée comme révélateur de situations sociales que nous pouvons qualifier d'exiliques et qui vont bien au-delà de l'expérience migratoire en elle-même. Ces expériences exiliques $s^{\prime} y$ avèrent intrinsèquement sociales, proches des définitions arendtiennes et weiliennes du déracinement. Elles se caractérisent par ailleurs par l'absence de concordance avec la migration, ainsi que par la possible et fréquente multiplicité, diversité et addition des causes et conditions exiliques chez un même individu. Et elles sont bien plus problématiques pour les personnages que leur corrélat géographique : elles s'avèrent l'expression d'une "violence symbolique " au sens bourdieusien, notamment

13 Dont le nom n'est pas prononcé, mais que l'on devine sous la métaphore de « La Verte " qui est aussi donnée à la capitale congolaise. 
discursive, exercée sur les individus et dont la force est soulignée par la profonde somatisation de certains personnages, attestant des conséquences à la fois psychologiques et physiologiques de ces situations exiliques. Tandis qu'elles traduisent la permanence de rapports raciaux hégémoniques et d'injonctions identitaires dans le pays de départ comme d'arrivée, les expériences exiliques interrogent surtout les limites du faire société.

Dans l'exemple précis du corpus choisi, la force heuristique de la littérature est révélée, aidant à formaliser cette violence symbolique et les modalités de ses intériorisations, à faire émerger des thèmes moins facilement exprimables, à dire des choses largement retranchées dans le domaine de l'indicible. Le discours des auteurs vient ainsi remettre en cause un certain ordre social, une certaine violence symbolique incorporée par les acteurs sociaux : $c^{\prime}$ est ainsi que I'on retrouve dans le pays d'origine comme en migration des individus dont la socialisation contient en germe la reproduction d'une domination extérieure et arbitraire. C'est par là, par exemple, que se fait jour la présence de relents évolutionnistes intégrés à la logique migrante. L'exilé est quant à lui celui qui se trouve rapporté à la marge dans une société donnée, qu'elle soit d'origine ou bien d'accueil dans le cas migratoire, et ici dans le cas franco-congolais encore largement dans le continuum d'une " situation coloniale " (Balandier, 1951) et par là encore, dans un contexte social adhérant encore largement au sens de cette violence symbolique. De ce type de violence dont Pierre Bourdieu soulignait qu'elle "s'inscrit durablement dans les corps des dominés, sous la forme de schèmes de perception et de dispositions "(Bourdieu, $1997: 245$ ), la littérature permet d'en dire les modalités. Par exemple, à cette forme de violence à laquelle est confronté Joseph lors de son retour - et à laquelle il résiste - s'oppose celle à laquelle adhère bientôt "Jo ", qui, par le truchement du Docteur Malfoi qui manipule en quelque sorte ses structures cognitives, se rend, renoncement auquel fait suite et durablement tout un ensemble de gestes d'obéissance et de soumission. Intégrer l'exil dans les études sur la migration permet ainsi de renforcer la compréhension des expériences migratoires et de comprendre des situations liminaires, des marginalisations, des expressions et conséquences de situations hégémoniques (Gramsci, 1975) et de violences symboliques et, par-là, de mieux comprendre l'individu migrant en interrogeant un continuum à la fois historique et social, individuel et subjectif. Enrichir les expériences migratoires d'une analyse des expériences exiliques permet alors de mieux articuler le collectif et l'individuel, l'objectif et le subjectif et surtout d'en interroger les dynamiques d'ouverture ou de fermeture, d'exclusion ou d'inclusion, d'ajustements, de négociations ou de blocages structurels.

Ainsi, si, dans L'Impasse, par exemple, la migration joue bien le rôle de révélateur de situations exiliques, elle n'est en réalité que le leurre d'un exil social et pour ainsi dire, transnational. Celui (voire ceux) vécu(s) au Congo se double(nt) d'un autre (voire d'autres), vécu(s) en France soit sous le signe du reniement de soi et de la défiance envers son passé et envers les siens - incarné par l'expérience de Joseph pendant les quinze ans passés en France sans retourner au Congo - soit sous le signe de l'exclusion et de l'injustice, dimension que soulignent notamment les personnages du Cour des enfants léopards de N'Sonde. Si l'on observe donc dans l'ensemble du corpus une multiplicité et une superposition pour chaque personnage de conditions exiliques proches des théories weiliennes et arendtiennes, Le Silence des esprits, surtout, souligne 
la diversité et l'universalité des expériences de l'exil - rejetant, comme dans Le Cour des enfants léopards, toute forme d'exotisme intrinsèque à celui-ci. L'exil n'est pas, chez aucun de ces auteurs, le propre du migrant. Par contre, si l'expérience migratoire peut apparaître, en tant que confrontation et ouverture sur l'Autre et sur l'Ailleurs, comme un prisme positif, elle peut en outre conduire à des situations exiliques, à l'image de celles vécues en France par les personnages de N'Sonde. Ainsi, dans L'Impasse, si l'expérience de l'exil vécue par Joseph le conduit au non-choix de la folie ou du renoncement à soi, de la mort (symbolique et sociale) ou du mensonge, l'expérience de la migration lui a ouvert les yeux. Car cette dernière est aussi celle de la confrontation et de la mise à mal d'un imaginaire migratoire ancré dans des logiques de domination d'un monde par un autre.

Biyoula souligne également l'impossibilité à être soi-même dans le retour et à réconcilier ce que Ricœur nomme identité " mêmeté " et "ipséité ": Joseph, de retour à Brazzaville, croule sous le poids des injonctions et des assignations résultant, d'une part, de sa condition d'homme africain à la peau jugée trop noire, et, d'autre part, de celle du migrant qu'il est devenu. L'exil creuse un peu plus son lit chez Joseph qui, petit à petit, s'enferme dans une posture de repli (Burgos, 1982) et s'aménage dans la folie et le renoncement un espace " autre " où apaiser son âme en l'endormant. Ce même processus d'exclusion puis d'aménagement d'espaces de repli dans la mort ou la folie est à observer chez N'Sonde. Les expériences exiliques représentées par ces auteurs peuvent se définir par l'impossibilité de se trouver une place " reconnue et garantie par les autres " selon les termes d'Arendt, tout en restant soi-même. Ainsi, souvent, les personnages mis en scène sont-ils à rapprocher des personnages liminaires que définit Scarpa (Scarpa, 2009).

Dans les romans de N'Sonde et Biyaoula, les personnages conscients de leurs conditions exiliques vont, au fur et à mesure des récits, être confrontés à l'incommunicabilité de ces expériences et n'auront alors pour exutoire que la folie, I'errance ou la mort - éventuellement symbolique... Ou de l'écriture, pour le personnage de Black Bazar au destin moins tragique, qui suggère en doublant, par sa propre rédaction du roman, le geste même des écrivains du corpus, que la littérature puisse apparaître comme un possible contournement de l'incommunicabilité des expériences exiliques. En effet, si les textes littéraires présentent le défaut apparent de l'élaboration et de l'absence de spontanéité, ils se départissent aussi en retour du cadre imposé par la relation d'enquête et finalement, produisent un discours " autre ", soumis à la nécessité de trouver les mots justes pour "nommer l'innommable " (Bonn, 2000), l'informulé des expériences vécues. Ainsi, l'aspect subjectif, et surtout introspectif, émerge plus aisément et agit comme le révélateur de situations incorporées et notamment ici, des expériences exiliques, de celles du déracinement weilien qui sont aussi celles d'une violence symbolique exercée sur les subjectivités.

L'analyse des ouvrages du corpus nous enjoint donc à reconnaître dans l'expérience de l'exil des ancrages politiques, mais aussi historiques et sociaux venant étendre la définition usuelle du terme d'exil. Questionner ainsi l'exil et ses expériences comme autant de faits sociaux à travers la littérature vient combler un impensé de l'approche des expériences migratoires en sciences sociales : la figure du migrant - et par là, la migration même - agit ici comme 
un révélateur des choses non résolues, des paradoxes, des mensonges et des non-dits de logiques se situant à la fois dans le continuum d'une certaine " situation coloniale " (Balandier, 1951) et dans le contexte de la mondialisation où de nouveaux imaginaires tout aussi déséquilibrés sont forgés, s'insérant alors aisément dans la multitude des flux matériels et idéels, mais aussi humains, qui la caractérisent.

\section{Références bibliographiques}

\section{Ouvrages universitaires}

Albert Christiane (2005) L'immigration dans le roman francophone contemporain, Paris, Karthala, 220 p.

Arendt Hannah (2005 [1951]) Les Origines du totalitarisme, Paris, Seuil, 380 p.

Balandier Georges (1951) La situation coloniale : approche théorique, Cahiers internationaux de sociologie, 11, pp. 44-79.

Barbour Sarah, Lacroix Thomas and Misrahi-Barak Judith (Eds.) (2015) Diasporas, Cultures of mobilities, "Races", vol. 2 Diasporas, Memory and Intimacy, Montpellier, Presses universitaires de la Méditerranée, 258 p.

Barrère Anne et Martuccelli Danilo (2009) Le roman comme laboratoire. De la connaissance littéraire à l'imagination sociologique, Villeneuve d'Asc, Septentrion, $373 \mathrm{p}$.

Bensa Alban et Pouillon François (2012) Terrains d'écrivains, Littérature et ethnographie, Toulouse, Anarchasis, $405 \mathrm{p}$.

Bianchi Olivia (2005) Penser l'exil pour penser l'être, Le Portique, 1, [en ligne] consulté le 26/11/2015. URL : http://leportique.revues.org/index519.html

Boltanski Luc (2004) La condition fotale, Paris, Gallimard, 420 p.

Bonn Charles (2000) L'exil et la quête d'identité, fausses portes pour une approche des littératures de l'émigration ?, communication du colloque Littérature maghrébine d'expression française entre clichés, lieux communs et originalité, Institut Bourguiba des langues vivantes, Tunis, 28-29 avril 2000, [en ligne]. URL : http://www.limag.refer.org/Textes/Bonn/EmigrTunisGafaiti.htm\#_ftn1

Bordes-Benayoun Chantal (2006) Introduction : I'exil, figure littéraire, figure sociologique, in William Berthomière et Christine Chivallon Dirs., Les diasporas dans le monde contemporain : un état des lieux, Paris-Pessac, Karthala-MSHA, pp. 189-194.

Bourdieu Pierre (1997) Méditations Pascaliennes, Paris, Le Seuil, 320 p.

Bourdieu Pierre (1986) L'lllusion biographique, Actes de la recherche en Sciences sociales, 62-63, pp. 69-72.

Brown Richard (1992) Clefs pour une poétique de la sociologie, Arles, Actes Sud, $351 \mathrm{p}$.

Burgi-Golub Noëlle (1999) D'exils en émotions, l'identité humaine, in Ragi Tariq Dir., Les territoires de l'identité, Paris, L'Harmattan, pp. 27-62.

Burgos Jean (1982) Pour une poétique de l'imaginaire, Paris, Seuil, 409 p. 
Candau Joël (1998) Mémoire et identité, Paris, PUF, 225 p.

Cassin Barbara (2013) La nostalgie. Quand donc est-on chez soi ? Ulysse, Énée, Arendt, Paris, Autrement, $148 \mathrm{p}$.

Cazenave Odile (2003) Afrique sur Seine, Une nouvelle génération de romanciers écrivains à Paris, Paris, L'Harmattan, $311 \mathrm{p}$.

Cnockaert Véronique, Privat Jean-Marie et Scarpa Marie (2011) L'Ethnocritique de la littérature (Anthologie), Québec, Presses de I'Université du Québec, 300 p.

Cohn Dorrit (1981) La Transparence intérieure, modes de représentation de la vie psychique dans le roman, Paris, Seuil, $310 \mathrm{p}$.

Copans Jean (2001) La situation coloniale de Georges Balandier : notion conjoncturelle ou modèle sociologique et historique, Cahiers internationaux de sociologie, $110(1)$, pp. 31-52.

De Gourcy Constance (2013) Partir, rester, habiter : le projet migratoire dans la littérature exilaire, Revue Européenne des Migrations Internationales, 29 (4), pp. 43-58.

Fouquet Thomas (2007) Imaginaires migratoires et expériences multiples de I'altérité : une dialectique actuelle du proche et du lointain, Autrepart, 41, pp. 83-97.

Gandoulou Justin-Daniel (1984) Entre Paris et Bacongo, Paris, Centre Georges Pompidou, $213 \mathrm{p}$.

Ghidina Jean-Igor et Violle Nicolas (2014) Récits de migration, en quête de nouveaux regards, Clermont-Ferrand, Presses universitaires Blaise Pascal, $330 \mathrm{p}$.

Goody Jack (2007) Pouvoirs et savoirs de l'écrit, Paris, La Dispute, 269 p.

Gramsci Antonio (1975) Gramsci dans le texte (1916-1935), Paris, Éditions sociales, $798 \mathrm{p}$.

Gras Pierre (Dir.) (2009) Exils/créations, quels passages ?, Paris, L'Harmattan, $90 \mathrm{p}$.

Grignon Claude et Passeron Jean-Claude (1989) Le Savant et le populaire Misérabilisme et populisme en sociologie et en littérature, Paris, Gallimard/Seuil, $260 \mathrm{p}$.

Heinich Nathalie (2005) Les limites de la fiction, L'Homme, 175-176, pp. 57-76.

Katambwe Jo M. (Dir.) (2011) Communication et lien social. Aux fondements de la sociabilité, Québec, Presses de l'Université Laval, 336 p.

Lahire Bernard (Dir.) (2011) Ce qu'ils vivent, ce qu'ils écrivent. Mises en, scènes littéraires du social et expériences socialisatrices des écrivains, Paris, Éditions des Archives Contemporaines, 573 p.

Lalagianni Vassiliki et Moura Jean-Marc (2014) Espace méditerranéen. Écritures de l'exil, migrances et discours postcolonial, Amsterdam, New York, Éditions Rodopi BV, 208 p.

Larangé Daniel S. (2014) De l'écriture africaine à la présence afropéenne. Pour une exploration de nouvelles terres littéraires, Paris, L'Harmattan, 241 p. 
Lepenies Wolf (1995) Les trois cultures. Entre science et littérature, l'évènement de la sociologie, Paris, Éditions de la Maison des Sciences de l'Homme, 408 p.

MacGaffey Wyatt (2000) Kongo political culture, The conceptual challenge of the particular, Bloomington and Indianapolis, Indiana University Press, 274 p.

Malkki Liisa H. (1995) Refugees and Exile: From "Refugee Studies" to the National Order of Things, Annual review of Anthropology, 24, pp. 495-523.

Martiniello Marco, Puig Nicolas et Suzanne Gilles (2009) Créations en migrations. Parcours, déplacements, racinements, Revue Européenne des Migrations Internationales, 25 (2), $147 \mathrm{p}$.

Misrahi-Barak Judith et Raynaud Claudine (2014) Diasporas, Cultures of mobilities, "Races", vol. 1 Diasporas and Cultures of Migrations, Montpellier, Presses Universitaires de la Méditerranée, $376 \mathrm{p}$.

Mongo-Mboussa Boniface (2002) La littérature des Africains de France, de la "Postcolonie " à l'immigration, Hommes \& migrations, 1239, pp. 67-74.

Montandon Alain (Dir.) (2006) Littérature et anthropologie, Nîmes, Société française de littérature générale et comparée (SFLGC), 338 p.

Montandon Alain et Pitaud Philippe (2006) Vieillir en exil, Clermont-Ferrand, Presses Universitaires Blaise Pascal, 210 p.

Moudileno Lydie (2006) Parades postcoloniales : la fabrication des identités dans le roman congolais, Paris, Karthala, $160 \mathrm{p}$.

Moudileno Lydie (2003) Littératures africaines francophones des années 1980 et 1990, Dakar, CODESRIA, 99 p.

Moudileno Lydie (2001) La fiction de la migration : manipulation des corps et des récits dans Bleu blanc rouge d'Alain Mabanckou, Présence Africaine, 1 (163-164), pp. 182-189.

Nouss Alexis (2015) La condition de l'exilé. Penser les migrations contemporaines, Paris, Éditions de la maison des Sciences de l'Homme, 175 p.

Nouss Alexis (Nuselovici) (2013) L'exil comme expérience, Séminaire EHESS/ Collège d'études mondiales : L'expérience de l'exil, Paris, EHESS, 20 février.

Pénicaud Mélanie (2017) Dire et écrire la migration, anthropologie de l'expression des expériences migratoires congolaises (Congo Brazzaville) vers la France, Thèse d'anthropologie, Université de Poitiers, $578 \mathrm{p}$.

Pénicaud Mélanie (2015) D'exils en migrations, échos d'une dispersion iranienne, in Sarah Barbour, Thomas Lacroix and Judith Misrahi-Barak Eds., Diasporas, Cultures of mobilities, "Races", vol. 2 Diasporas, Memory and Intimacy, Montpellier, Presses universitaires de la Méditerranée, pp. 33-50.

Pénicaud Mélanie (2012) Territoires de soi, parole d'exils. Approche comparative des modes d'expression de l'expérience migratoire des élites iraniennes en France, Mémoire de master II, Poitiers, Université de Poitiers, 140 p.

Privat Jean-Marie (1994) Bovary Charivari. Essai d'ethno-critique, Paris, CNRS Éditions, 314 p.

Ricœur Paul (1997) L'Idéologie et l'utopie, Paris, Seuil, 432 p. 
Ricœur Paul (1990) Mimésis, référence et refiguration dans Temps et récits, Études phénoménologiques, 11 (4), pp. 29-40.

Saïd Edward W. (2002) Reflections on Exile and Other Essays, Cambridge, Harvard University Press, $656 \mathrm{p}$.

Scarpa Marie (2009) Le personnage liminaire, Romantisme, 145 (3), pp. 25-35.

Schor Ralph (2013) Écrire en exil, Les écrivains étrangers en France, Paris, CNRS éditions, $342 \mathrm{p}$.

Simon-Lorière Hélène (2013) Conditions de vie et projets migratoires des réfugiés libériens à Conakry (Guinée) et Accra (Ghana), Thèse de géographie, Université de Poitiers, $652 \mathrm{p}$.

Soret Marcel (2005) Les Kongo nord-occidentaux, Paris, L'Harmattan, 144 p.

Talahite-Moodley Anissa (2007) Problématiques identitaires et discours de l'exil dans les littératures francophones, Ottawa, Presses de I'Université d'Ottawa, $380 \mathrm{p}$.

Thomas Dominic (2013) Noirs d'encre. Colonialisme, immigration et identité au cœur de la littérature afro-française, Paris, La Découverte, 297 p.

Weil Simone (1949) L'enracinement, Paris, Gallimard, 381 p.

Wievorka Michel (2013) Pour un concept d'exil, Séminaire EHESS/Collège d'études mondiales : L'expérience de l'exil, Paris, EHESS, 20 février.

\section{Cuvres littéraires}

Biyaoula Daniel (1996) L'Impasse, Paris, Présence africaine, $327 \mathrm{p}$.

Mabanckou Alain (2009) Black Bazar, Paris, Seuil, 265 p.

N'Sonde Wilfried (2010) Le silence des esprits, Arles, Actes Sud, 170 p.

N'Sonde Wilfried (2007) Le cœur des enfants léopards, Arles, Actes Sud, 132 p. 


\section{Mélanie Pénicaud}

\section{Expérience migratoire et exil social dans la migration congolaise : de l'enjeu d'un corpus littéraire en sciences sociales. Biyaoula, Mabanckou et N'Sonde}

Cet article s'appuie sur l'analyse d'œuvres littéraires d'auteurs d'origine congolaise ayant vécu en France pour interroger la migration comme un possible espace d'une convergence disciplinaire entre littérature et sciences sociales. II conduit à faire l'hypothèse d'un exil de nature sociale, par opposition à l'idée doxatique et restrictive d'un exil synonyme d'une " migration forcée ". Dans ce contexte, I'expérience exilique du Congolais en Afrique et en Europe se situe d'abord dans le continuum d'une " situation coloniale " et se creuse encore dans la migration en France, par le biais d'une marginalisation et d'une violence symbolique, notamment discursive, exercées sur les immigrés au sein de l'Étatnation. L'utilisation d'un corpus littéraire dans une perspective anthropologique conduit à interroger la communicabilité des expériences exiliques et par là, à affiner la compréhension des expériences migratoires tout en explorant diverses pistes théoriques et méthodologiques.

\section{Migratory Experience and Social Exile in the Congolese Migration: Of Using a Literary Corpus in Social Sciences. Biyaoula, Mabanckou and N'Sonde}

This article is based on an analysis of literary works written by Congolese authors who have lived in France to consider migration as a possible space for meeting points between literature and social sciences. This leads to hypothesize a social nature of exile in contrast with the dogmatic and restrictive idea seen as a "forced migration". In this context, the Congolese's exile experience in Africa and Europe lies first in a continuum of the French "colonial situation" and is strengthened in case of migration to France, through marginalization and symbolic violence, mainly discursive, exerted on immigrants inside the Nation-State. The use of a literary corpus from an anthropological angle leads to question the communicability of exile experiences and hence to refine the comprehension of migratory experiences while exploring varied theoretical and methodological tracks.

\section{Experiencia migratoria y exilio social en la migración congoleña: del desafío de un corpus literario en ciencias sociales. Biyaoula, Mabanckou y N'Sonde}

Este artículo se apoya en el análisis de obras literarias de autores de origen congoleño que han vivido en Francia para cuestionar la migración como posible espacio de convergencia disciplinario entre literatura y ciencias sociales. Conduce a plantear la hipótesis de una naturaleza social del exilio, al contrario de la idea doxástica y restrictiva de un exilio sinónimo de "migración forzada». En este contexto, la experiencia exílica del congoleño en África y en Europa se sitúa primero en la continuidad de una situación colonial que sigue creciendo en la migración en Francia, mediante una marginación y de una violencia simbólica, especialmente discursiva, que se ejerce sobre los inmigrantes en el seno del estado nación. El uso de un corpus literario para antropología conduce a cuestionar la comunicatividad de las experiencias exílicas y con eso, afinar la comprensión de las experiencias migratorias a la vez que se exploran diversas pistas teóricas y metodológicas. 